\title{
Direct Numerical Simulation of the turbulent natural convection flow in an open cavity of aspect ratio 4
}

\author{
J. Chiva ${ }^{1}$, O. Lehmkuhl ${ }^{1,2}$, R. Borrell ${ }^{1,2}$ and A. Oliva ${ }^{1}$ \\ ${ }^{1}$ Heat and Mass Transfer Technological Center, Polytechnical University of Catalonia (UPC) \\ Colom 11,08222,Terrassa, Barcelona,Spain.cttc@cttc.upc.edu \\ ${ }^{2}$ Termo Fluids, S.L., Magí Colet, 8, 08204 Sabadell (Barcelona), Spain
}

\begin{abstract}
In this paper, three-dimensional turbulent natural convection heat transfer in an open cavity with an isothermal wall facing the overture has been studied. The aspect ratio chosen for the cavity has been 4 to complement the studies by Trias et al. [1,2] of closed cavities with the same aspect ratio. Direct numerical simulations (DNS) of the cavity are presented and analyzed. Rayleigh numbers up to $R a=10^{12}$ has been considered.
\end{abstract}

\section{Introduction}

Natural convection heat transfer in cavities has been studied extensively in the literature due to its relevance to many engineering areas such as low temperature solar collectors, design of buildings, cooling of nuclear reactors, etc. However, the majority of the studies are devoted to the behavior of closed cavities. To mention a few: De Vahl Davis [3] provided a well known set of benchmark solutions for steady natural convection of air in a horizontally heated square cavity for Rayleigh numbers up to $R a=10^{6}$, Le Quéré [4] extended the analysis up to $R a=10^{8}$, Trias et al. [1,2] computed direct numerical simulations (DNS) of two- and three-dimensional turbulent natural convection flows in a differentially heated cavity of aspect ratio 4 for Rayleigh numbers up to $R a=10^{10}$. Later, Trias et al. [5, 6] (in two papers) extended this study for Rayleigh numbers up to $R a=10^{11}$. Chenoweth and Paolucci [7] investigated the steadystate flow in rectangular cavities with large temperature differences between vertical isothermal walls. They used the transient form of the flow equations, simplified for low-Mach number flows. Vierendeels et al. [8] presented a benchmark for the laminar natural convective heat transfer problem in a square cavity with large horizontal temperature differences.

For the case of open cavities, there is a lack of studies in the literature which analyses the 3D turbulent flow present at high temperature differences. This scenery is present in many industrial applications such as solar power towers, dish stirling systems, electronic components, etc.. The fact that the cavity is open makes the boundary conditions more complex and the domain has to be extended to include part of the external environment in order to avoid any interaction between the boundary conditions and the flow inside the cavity (Juárez et al. [9]).

In the present paper, the turbulent natural convection of air in an open cavity of aspect ratio 4, Rayleigh number based on the height of the cavity (H2) $10^{12}$ and Prandtl number $\operatorname{Pr}=0.71$, is studied by means of DNS. The main characteristics of the instantaneous turbulent flow, together with the time-averaged flow are discussed in detail.

\section{Mathematical formulation}

In all the DNS studies presented in this paper the following conservative formulation adapted to unstructured meshes have been used. If we write the discretised Navier-Stokes, continuity and 
energy equations, assuming the Boussinesq approximation, as

$$
\begin{aligned}
\mathrm{M} \boldsymbol{u} & =\mathbf{0} \\
\rho \frac{\partial \boldsymbol{u}}{\partial t}+\mathrm{C}(\boldsymbol{u}) \boldsymbol{u}+\mathrm{D} \boldsymbol{u}+\mathrm{G} \boldsymbol{p}+f & =\mathbf{0} \\
\frac{\partial T}{\partial t}+\mathrm{C}(\boldsymbol{u}) T+\alpha \mathrm{D} T & =\mathbf{0}
\end{aligned}
$$

where $\boldsymbol{u} \in \mathbb{R}^{3 m}, \boldsymbol{p} \in \mathbb{R}^{m}$ and $\boldsymbol{T} \in \mathbb{R}^{m}$ are the velocity vector, pressure and temperature, respectively (here $\mathrm{m}$ applies for the total number of control volumes $(\mathrm{CV})$ of the discretised domain), $\nu$ is the kinematic viscosity, $\rho$ the density and $\alpha$ the thermal diffusivity. $f$ is the body force $f=\beta\left(T-T_{c}\right) g$, where $\beta$ is the thermal expansion coefficient, $T_{c}$ is the cold temperature and $g$ is the gravity. Convective and diffusive operators in the momentum equation for the velocity field are given by $\mathrm{C}(\boldsymbol{u})=(\boldsymbol{u} \cdot \nabla) \in \mathbb{R}^{3 m \times 3 m}, \mathrm{D}=-\nabla^{2} \in \mathbb{R}^{3 m \times 3 m}$ respectively. Gradient and divergence (of a vector) operators are given by $\mathrm{G}=\nabla \in \mathbb{R}^{3 m \times m}$ and $\mathrm{M}=\boldsymbol{\nabla} \cdot \epsilon$ $\mathbb{R}^{m \times 3 m}$ respectively.

The governing equations used in TermoFluids [10] have been discretised on a collocated unstructured grid arrangement by means of second-order spectro-consistent schemes. Such schemes are conservative, i.e. they preserve the kinetic energy equation. These conservation properties are held if, and only if the discrete convective operator is skew-symmetric $(C(\boldsymbol{u})=$ $-C^{*}(\boldsymbol{u})$ ), the negative conjugate transpose of the discrete gradient operator is exactly equal to the divergence operator $\left(-(\Omega G)^{*}=M\right)$ and the diffusive operator $D$, is symmetric and positivedefinite. These properties ensure both, stability and conservation of the kinetic-energy balance even at high Reynolds numbers and with coarse grids (Verstappen and Van Der Velde [11]).

For the temporal discretization of the momentum equation, a two-step linear explicit scheme on a fractional-step method has been used, while for the pressure-gradient term an explicit firstorder scheme has been used. The velocity-pressure coupling has been solved by means of a classical fractional step projection method,

$$
\boldsymbol{u}^{p}=\boldsymbol{u}^{n+1}+\mathrm{G} \tilde{\boldsymbol{p}}
$$

where $\tilde{\boldsymbol{p}}=\boldsymbol{p}^{n+1} \Delta t^{n} / \rho$ is the pseudo-pressure, $\boldsymbol{u}^{p}$ the predicted velocity, $n+1$ is the instant where the temporal variables are calculated, and $\Delta t^{n}$ is the current time step $\left(\Delta t^{n}=t^{n+1}-t^{n}\right)$. Taking the divergence of (4) and applying the incompressibility condition yields a discrete Poisson equation for $\tilde{\boldsymbol{p}}$ : $\mathrm{L} \tilde{\boldsymbol{p}}=\mathbf{M} \boldsymbol{u}^{p}$. The discrete laplacian operator $\mathrm{L} \in \mathbb{R}^{m \times m}$ is, by construction, a symmetric positive definite matrix $\left(\mathrm{L} \equiv \mathrm{M} \Omega^{-1} \mathrm{M}^{*}\right)$. Once the solution of $\boldsymbol{p}^{n+1}$ is obtained, $\tilde{\boldsymbol{p}}$ results from equation 4 . Finally the mass-conserving velocity at the faces $\left(\mathrm{M}_{s} \boldsymbol{u}_{s}^{n+1}=0\right)$ is obtained from the correction,

$$
\boldsymbol{u}_{s}^{n+1}=\boldsymbol{u}_{s}^{p}-\mathrm{G}_{s} \tilde{\boldsymbol{p}}
$$

where $\mathrm{G}_{s}$ represents the discrete gradient operator at the $\mathrm{CV}$ faces. This approximation allows to conserve mass at the faces but it has several implications. If the conservative term is computed using $\boldsymbol{u}_{s}^{n+1}$, in practice an additional term proportional to the third-order derivative of $\boldsymbol{p}^{n+1}$ is introduced. Thus, in many aspects, this approach is similar to the popular Rhie \& Chow interpolation method and eliminates checkerboard modes.

However, collocated meshes do not conserve the kinetic energy. When the fractional step method on a collocated arrangement is used, there are two sources of errors in the kinetic energy conservation: i) due to interpolation schemes and, ii) due to inconsistency in the pressure field 
in order to ensure mass conservation. While the first one can be eliminated through the use of conservative schemes such as those used in the present work, the latter equals to:

$$
\epsilon_{\mathbf{k e}}=(\tilde{\boldsymbol{p}})^{*} \mathrm{M}\left(\mathrm{G}-\mathrm{G}_{s}\right) \tilde{\boldsymbol{p}}
$$

This contribution of the pressure gradient term to the evolution of the kinetic energy can not be eliminated. It can be shown that the spatial term of the pressure error scales as $O\left(\Delta x^{2}\right)$ and the temporal term scales as $O(\Delta t)$, i.e. pressure errors are of the order of $O\left(\Delta x^{2} \Delta t\right)$. However, the pressure errors do not have a significant impact on the results at grid resolutions and time-steps used in LES and in DNS.

\section{Problem definition and numerical domain}

The situation under consideration in this work is natural convection of air $(\operatorname{Pr}=0.71)$ in an open cavity (see Figure 1) with a Rayleigh number, based on the height of the cavity (H2), of $R a=10^{12}$.

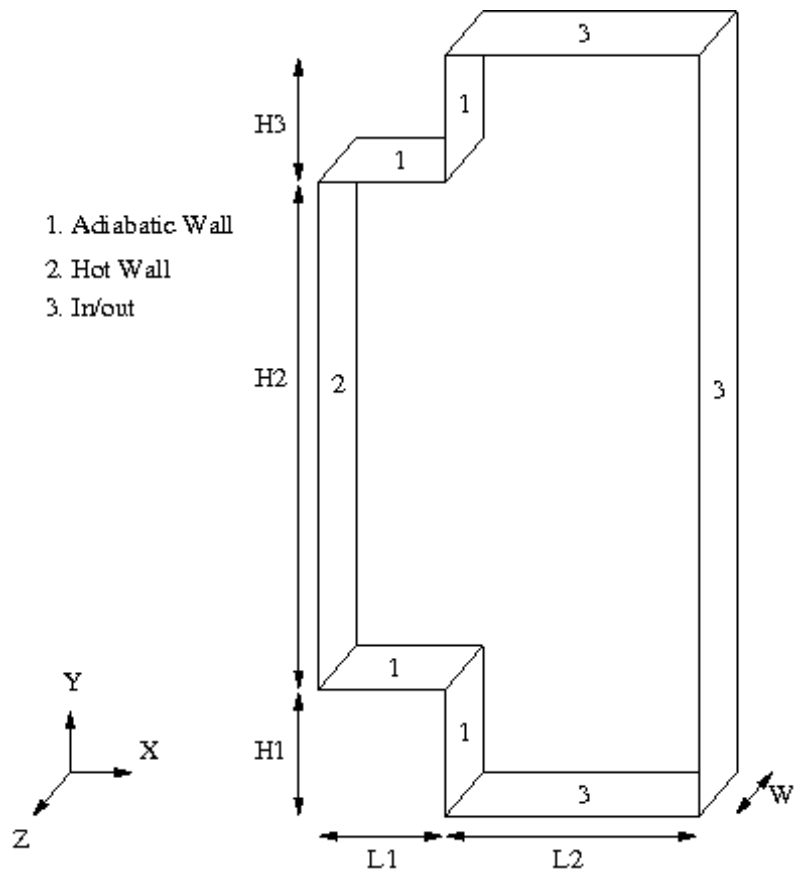

Figure 1: Scheme of the open cavity.

The vertical hot wall (identified with 2 in Figure 1) is considered isothermal with temperature $T_{h}$. The top, bottom and surfaces attached to them (corresponding with boundary 1 in Figure 1) are considered adiabatic. No slip conditions are set at the solid walls. The flow has been considered periodic in the $\mathrm{z}$ axis. Pressure boundary conditions are imposed at the top, bottom and left boundaries of the domain (boundary 3 in Figure 1), with Neumann condition for temperature if the air goes out of the system and temperature $T_{c}$ if it goes in.

In Table 1 the characteristics aspect ratios of the cavity are specified. Considering the reference scales for length, time, velocity, temperature and dynamic pressure as $H 2,\left((H 2)^{2} / \alpha\right) R a^{-0.5}$, $(\alpha / H 2) R a^{0.5}, T_{h}-T_{c}, \rho\left(\alpha /(H 2)^{2}\right) R a$, respectively, thermal convection in the cavity is governed by the Rayleigh number $\left(R a=g \beta\left(T_{h}-T_{c}\right)(H 2)^{3} \operatorname{Pr} / \nu^{2}\right.$, Prandtl number $(\operatorname{Pr}=\nu / \alpha)$ and height aspect ratio $H 2 / L 1$. 
Table 1: Characteristic aspect ratios of the cavity.

\begin{tabular}{ll}
\hline Aspect ratio & Value \\
\hline H2/L1 & 4 \\
H1/L1 & 1 \\
H3/L1 & 1 \\
L2/L1 & 2 \\
W/L1 & 0.5 \\
\hline
\end{tabular}

The meshes used for solving the domain considered have been generated by a constant step extrusion of a two-dimensional (2D) structured grid. Under these conditions, the spanwise coupling of the discrete Poisson equation, which results from the incompressibility constrain, yields circulant sub-matrices that are diagonalizable in a Fourier space. This allows to solve the Poisson equation by means of a FastFourier Transform (FFT) method. The algorithm used is based on the explicit calculation and direct solution of a Schur Complement system for the independent 2D systems. For more details the reader is referred to [12].

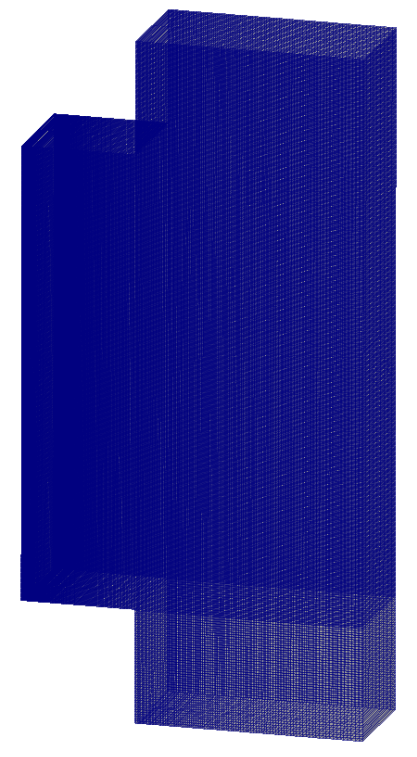

(a)

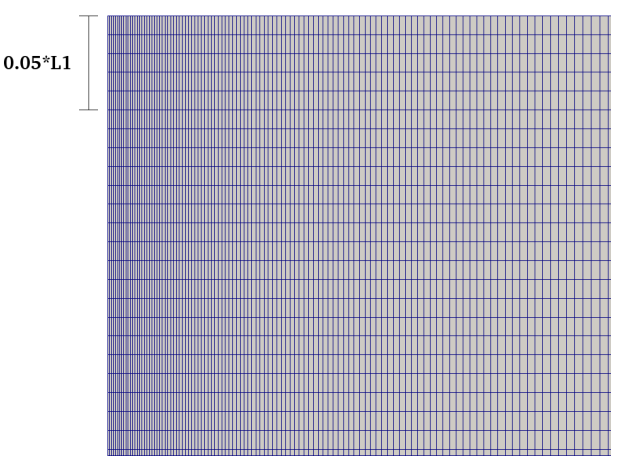

(b)

Figure 2: (a) 3D Mesh and (b) 2D detail of the mesh at the corner between the hot wall and the top adiabatic wall.

The number of control volumes of the final mesh has been 145.506 by 64 planes (about 9.3 millions of control volumes). In order to properly evaluate the boundary layer near the hot wall, a stretching has been applied to concentrate the maximum number of control volumes inside the boundary layer. In Figure 2(b) a detail of the mesh size at the top corner is shown.

\section{Results}

The simulation has been started with an air temperature inside the cavity $(x / L 1=0-1$, $y / L 1=0-4$ and $z / L 1=0-0.5)$ of $0.5\left(T_{h}-T_{c}\right)$ and the temperature of the remaining 
domain $(x / L 1=1-3, y / L 1=-1-5$ and $z / L 1=0-0.5)$ has been set to $T_{c}$. This configuration helps the simulation generating a huge plume of air that goes up and out of the cavity, obtaining a velocity field where air enters the system from the bottom of the domain and gets out from the top. Then, the simulation has been advanced in time until statistical stationary flow conditions have been achieved. After the initial transient has been completely washed out, averaged statistics have been computed over 124 time units.

\subsection{Instantaneous flow}

The resolution of the grid allows the visualization of very small scale 3D structures, as can be seen Figure 3, where instantaneous profiles of the temperature field are depicted. Next to the hot wall, the ascension of thermal plumes which produce a small recirculation at the top corner of the cavity can be observed. There is an entrainment of cold fluid from the bottom of the cavity which produces a recirculation which seem to be close to a quarter of the total height of the cavity (H2). The interaction between this cold fluid entrainment with the hot wall produces fluid instabilities at the bottom of the boundary layer, helping the generation of turbulence.

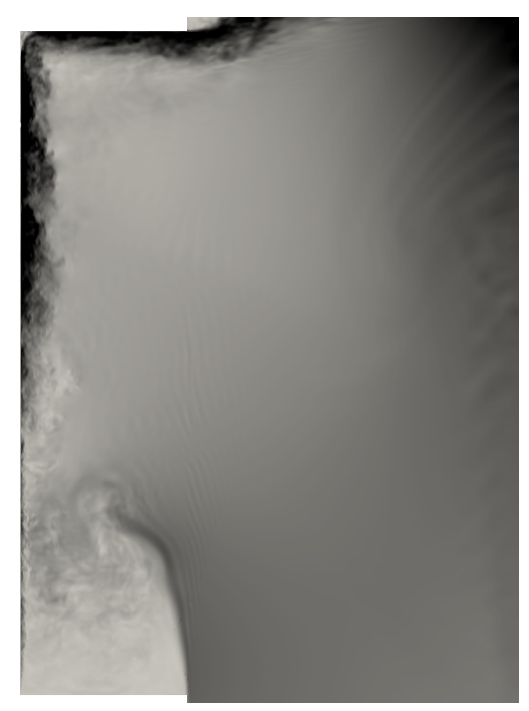

(a)

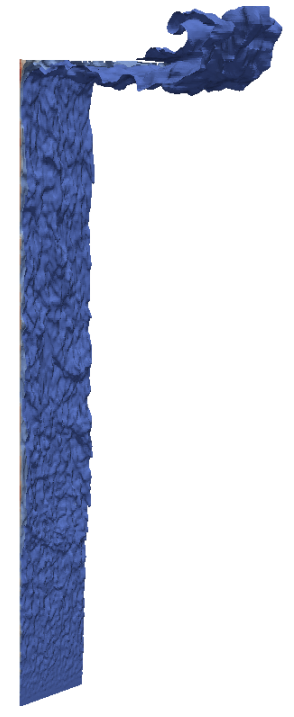

(b)

Figure 3: Instantaneous snapshots: (a) 2D view at the symmetry plane of the magnitude of the velocity and (b) $3 \mathrm{D}$ contours of the thermal field.

A more detailed analysis of these small scale structures can be performed by analysis of the coherent structures in the instantaneous flow. There are several techniques for the identification of the coherent structures of the flow (see for instance $[13,14]$ ). In the present study, the $Q$ criterion proposed by Hunt et al. [13] has been used. This method is based on the second invariant of the velocity gradient tensor $\nabla \boldsymbol{u}$. The velocity gradient tensor can be decomposed into a symmetric (rate-of-strain, $\mathrm{S}$ ) and a skew-symmetric (rate-of-rotation, $\Omega$ ) components. The second invariant of $\nabla \boldsymbol{u}$ is,

$$
Q=\frac{1}{2}\left(\|\boldsymbol{\Omega}\|^{2}-\|\boldsymbol{S}\|^{2}\right)
$$

where $\|\boldsymbol{\Omega}\|^{2}$ and $\|\boldsymbol{S}\|^{2}$ are the trace of the skew-symmetric and symmetric components of $\nabla \boldsymbol{u}$. This method defines an eddy structure as a region with positive second invariant $(Q)$, i.e. regions 
where rotation dominates over strain. In Figure 4, coherent structures for a value of $Q=0.3$ are depicted. The details of the top and bottom regions show that very small scale 3D structures can be properly simulated due to the resolution of the grid chosen. This small structures are generated very close to the base of the hot wall by the fluid instabilities produced by the aforementioned recirculation in this zone.

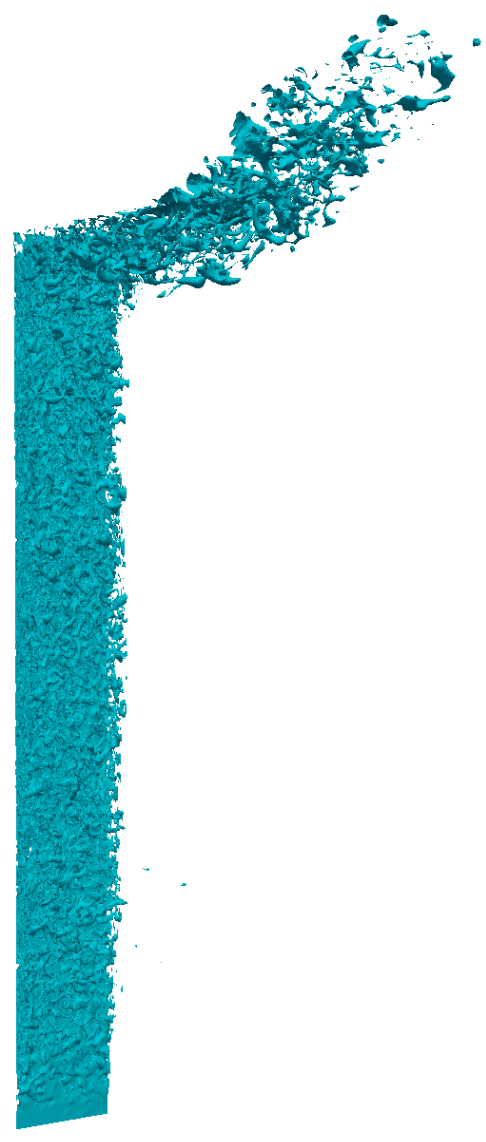

(a)

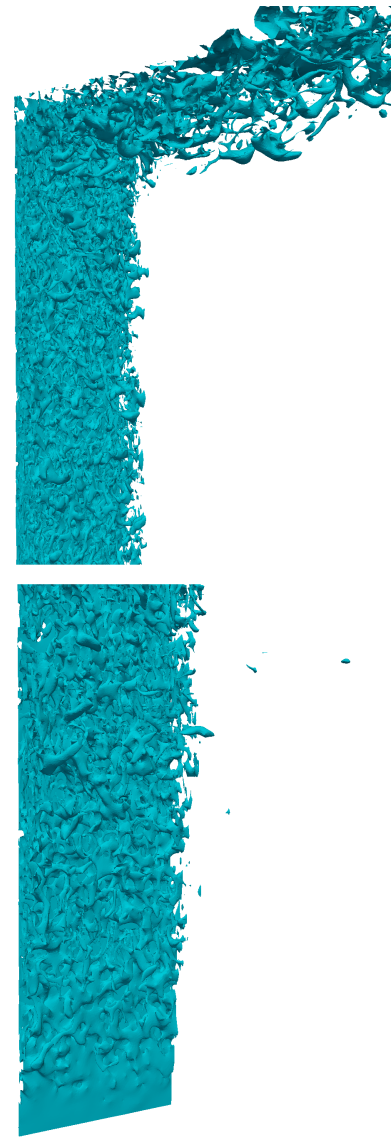

(b)

Figure 4: Instantaneous coherent structures for $Q=0.3$ (a) whole cavity (b) details of the top and bottom of the cavity.

\subsection{Time-averaged statistics}

Time-averaged statistics present in this paper have been obtained by averaging in time and homogeneous direction the computed variables.

The time-averaged stream lines, temperature contours, distribution of temperature variance and turbulent kinetic energy are depicted in Figure 5. The recirculation between the hot wall and the bottom of the cavity observed from the analysis of the instantaneous flow is clearly presented by the stream lines in Figure 5(a), with center at $x / L 1=0.465$ and $y / L 1=0.353$. This recirculation propitiates a stratification of the fluid temperature in this zone (see Figures 5(b) and 5(c)) and creates 3D structures near the bottom of the hot wall, producing turbulent kinetic energy in the near wall region. This turbulent kinetic energy is convected upwards by the thermal plumes (see Figure 5(d)). 


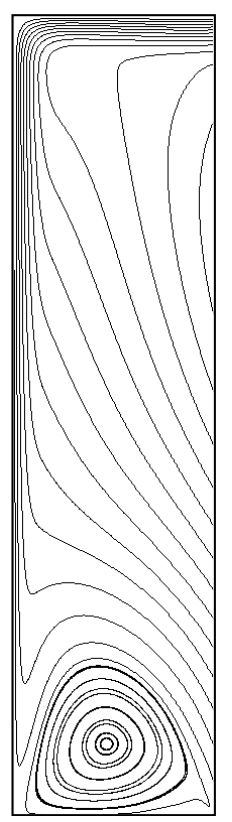

(a)

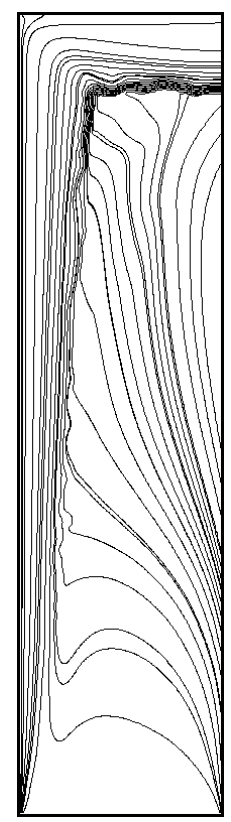

(b)

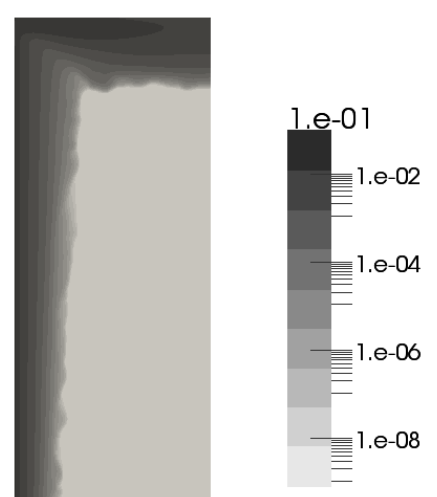

$1 . \overline{\mathrm{e}-10}$

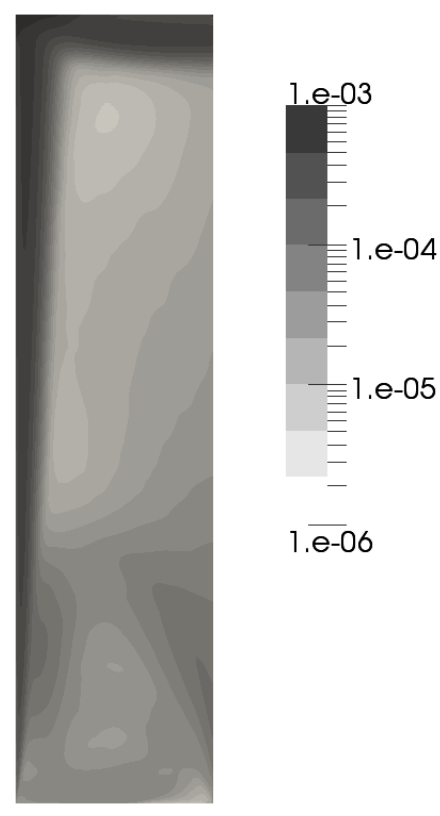

(d)

Figure 5: Time-averaged statistics in the cavity. (a) stream lines, (b) temperature contours, (c) distribution of temperature variance and (d) turbulent kinetic energy.

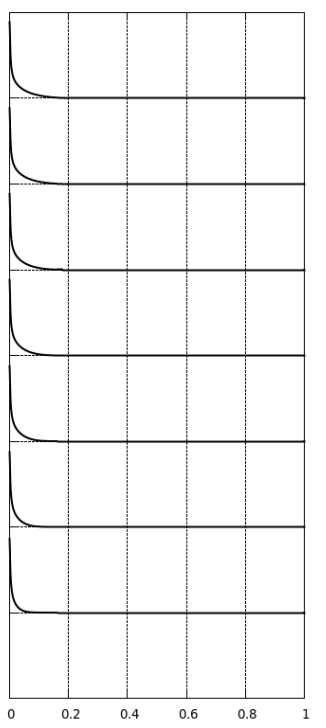

(a)

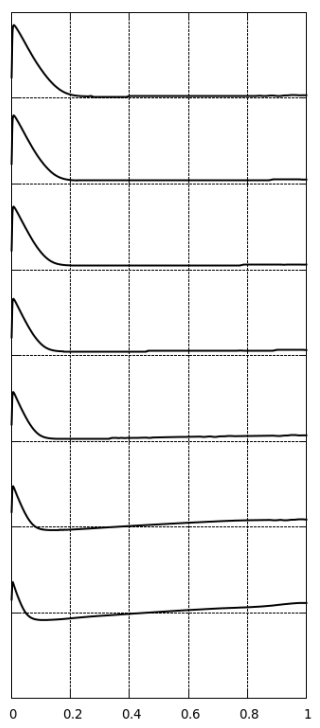

(b)

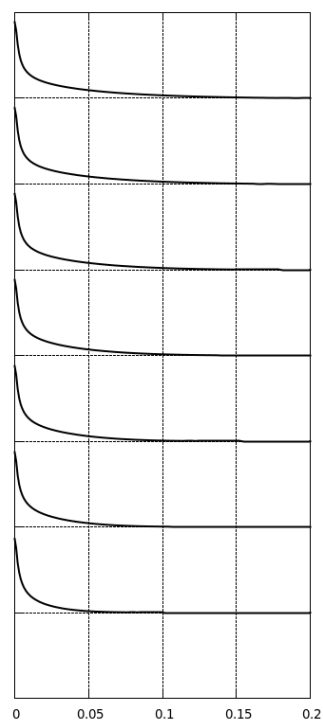

(c)

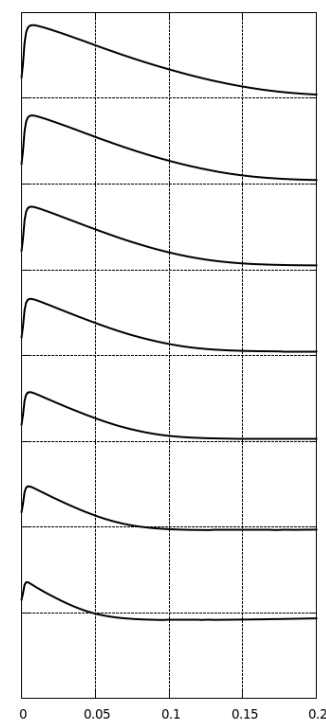

(d)

Figure 6: Time-averaged statistics at different heighs in the cavity. (a) temperature and (b) vertical velocity profiles at $y / L 1=0.5,1,1.5,2,2.5,3,3.5$ for $x / L 1=0-1$ and detail near the hot wall $(x / L 1=0-0.2)(\mathrm{c})(\mathrm{d})$. Each vertical subdivision represents 1 unit for temperature and 0.15 units for vertical velocity.

In Figure 6 time-averaged temperature and vertical velocity profiles are plotted for different heights $(y / L 1)$. In the left side, Figures (6(a) and 6(b) show the whole domain of the cavity 
$(x / L 1=0-1)$, whereas in the right side, Figures 6(c) and 6(d) depict more detailed versions $(x / L 1=0-0.2)$ of the left side ones. It can be seen that the vertical velocity profiles for the first 2 positions $(y / L 1=0.5$ and $y / L 1=1)$ have negative values between the boundary layer and the center of the cavity. This is caused by the aforementioned fluid entrainment at the bottom of the cavity. The flow moves faster as it advances along the hot wall to the top, while the thermal boundary layer gets wider.

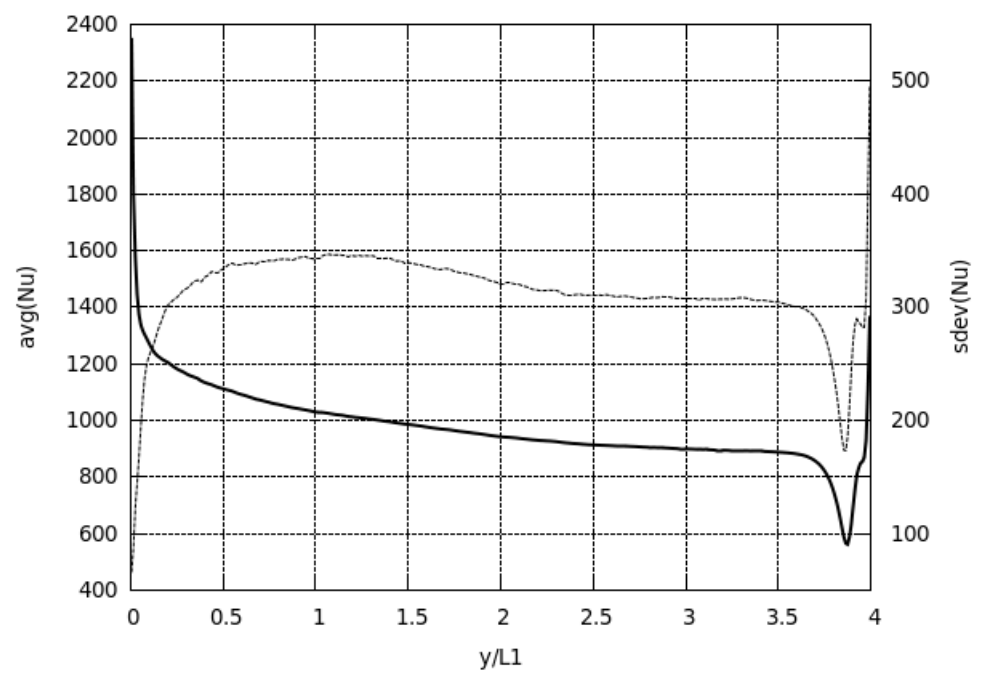

Figure 7: Profile of the averaged Nusselt number along the hot wall (solid lines) and its standard deviation (dashed lines).

In Figure 7 the profiles of the averaged Nusselt number along the hot wall and its standard deviation are shown. The overall averaged Nusselt number is 978. Comparing the present results with the work of Trias et al. [6] for the case of a closed cavity of aspect ratio 4 with $R a=10^{11}$, it could be noted that in the case of study of this work there is no transition point between laminar and turbulent regime, which in the case of Trias et al. occurs close to mid height. This is because in the present work the flow is fully turbulent from the beginning due to the influence of the fluid entrainment from the bottom of the cavity. Near the top of the cavity the Nusselt number manifests the behavior of an impinging phenomena, where the temperature of the air gets closer to the temperature of the wall due to the collision of the vertical flow against the top of the cavity.

\section{Conclusions and future actions}

The turbulent natural convection of air in an open cavity of aspect ratio 4 and $R a=10^{12}$ has been studied by means of DNS. Instantaneous flow and the time averaged statistics have been presented and analyzed. The flow has been found to be 3D and fully turbulent along the hot wall due to the fluid instabilities caused by the recirculation at the bottom of the cavity.

In the future, we will continue this work with simulations of the same cavity but with variable thermo-physical properties to analyze the validity of the Boussinesq approximation.

\section{Acknowledgements}

This work has been financially supported by the Ministerio de Educación y Ciencia, Secretaría de Estado de Universidades e Investigación, Spain (ref. ENE2009-09496 and ref. ENE2010- 
17801) and by the Collaboration Project between Universidad Politècnica de Catalunya and Termo Fluids S.L.

\section{References}

1. F.X. Trias, M. Soria, C.D. Pérez-Segarra and A. Oliva, Direct numerical simulation of a three-dimensional natural convection flow in a differentially heated cavity of aspect ratio 4, Numerical Heat Transfer part A-Applications 45 (2004) 649-673.

2. F.X. Trias, M. Soria, C.D. Pérez-Segarra and A. Oliva, Direct numerical simulations of two and three dimensional turbulent natural convection flows in a differentially heated cavity of aspect ratio 4, Journal of Fluid Mechanics 586 (2007) 259-293.

3. G. de Vahl Davis, Natural convection of air in a square cavity: a bench mark numerical solution, Int. J. Numer. Methods Fluids 3 (1983) 227-248.

4. P. Le Quéré, Accurate solutions to the square thermally driven cavity at high Rayleigh number, Comput. Fluids 20 (1991) 29-42.

5. F.X. Trias, A. Gorobets, M. Soria and A. Oliva, Direct numerical simulations of a differentially heated cavity of aspect ratio 4 with Rayleigh numbers up to $10^{11}$-Part I: Numerical methods and time-averaged flow, International Journal of Heat and Mass Transfer 53 (2010) 665-673.

6. F.X. Trias, A. Gorobets, M. Soria and A. Oliva, Direct numerical simulations of a differentially heated cavity of aspect ratio 4 with Rayleigh numbers up to $10^{11}$-Part II: Heat transfer and flow dynamics, International Journal of Heat and Mass Transfer 53 (2010) 674-683.

7. D.R. Chenoweth, S. Paolucci, Natural convection in an enclosed vertical air layer with large horizontal temperature differences, Journal of Fluid Mechanics 169 (1986) 173-210.

8. J. Vierendeels, B. Merci, E. Dick, Benchmark solutions for the natural convective heat transfer problem in a square cavity with large horizontal temperature differences, International Journal of Numerical Methods for Heat and Fluid Flow 13 (8) (2003) 1057-1078.

9. J.O. Juárez, J.F. Hinojosa, J.P. Xamán and M. Pérez-Tello, Numerical study of natural convection in an open cavity considering temperature-dependent fluid properties, International Journal of Thermal Sciences 50 (11) (2011) 2184-2197.

10. O. Lehmkuhl, C. D. Pérez Segarra, R. Borrell, M. Soria and A. Oliva. TermoFluids: A new Parallel unstructured CFD code for the simulation of turbulent industrial problems on low cost PC Cluster, Proceedings of the Parallel CFD 2007 Conference, pages 1-8.

11. R.W.C.P. Verstappen and R.M. Van Der Velde, Symmetry-Preserving Discretization of Heat Transfer in a Complex Turbulent Flow, Journal of Engineering Mathematics 54 (4) (2006) 299-318.

12. R. Borrell, O. Lehmkuhl, F. Trias and A. Oliva. Parallel direct Poisson solver for discretisations with one Fourier diagonalisable direction, Computational Physics, Vol. 230, No. 12, 2011, pp. 4723-4741.

13. J.C.R. Hunt, A.A. Wray and P. Moin. Eddies, stream and convergence zones in turbulent flows, Technical Report CTR-S88, Center for turbulent research (1988).

14. J. Jeong and F. Hussain. On the identification of a vortex, Journal of Fluid Mechanics 285 (1995) 69-94. 